\title{
Article
}

\section{From $F=m a$ to Flying Squirrels: Curricular Change in an Introductory Physics Course}

\author{
Brian O’Shea, ${ }^{*}{ }^{\dagger}$ Laura Terry, ${ }^{\ddagger}$ and Walter Benenson*
}

\author{
${ }^{*}$ Lyman Briggs College and ${ }^{\dagger}$ Department of Physics and Astronomy, Michigan State University, East Lansing, \\ MI 48825; ‡College of Education, Michigan State University, East Lansing, MI 48824
}

Submitted September 1, 2012; Revised March 12, 2013; Accepted March 12, 2013

Monitoring Editor: Eric Brewe

\begin{abstract}
We present outcomes from curricular changes made to an introductory calculus-based physics course whose audience is primarily life sciences majors, the majority of whom plan to pursue postbaccalaureate studies in medical and scientific fields. During the 2011-2012 academic year, we implemented a Physics of the Life Sciences curriculum centered on a draft textbook that takes a novel approach to teaching physics to life sciences majors. In addition, substantial revisions were made to the homework and hands-on components of the course to emphasize the relationship between physics and the life sciences and to help the students learn to apply physical intuition to life sciences-oriented problems. Student learning and attitudinal outcomes were assessed both quantitatively, using standard physics education research instruments, and qualitatively, using student surveys and a series of postsemester interviews. Students experienced high conceptual learning gains, comparable to other active learning-based physics courses. Qualitatively, a substantial fraction of interviewed students reported an increased interest in physics relative to the beginning of the semester. Furthermore, more than half of students self-reported that they could now relate physics topics to their majors and future careers, with interviewed subjects demonstrating a high level of ability to come up with examples of how physics affects living organisms and how it helped them to better understand content presented in courses in their major.
\end{abstract}

\section{INTRODUCTION}

Experienced practitioners of medicine and the life sciences are keenly aware of the role that physics plays in the structures and behaviors of living systems at all levels, from molecules to ecosystems. Beginning students of these subjects, however, have a very difficult time connecting the topics that they learn in a typical introductory physics course sequence to their chosen college major and career path-a source of great frustration to their instructors! This disconnect is only becoming more critical as life scientists rely more heavily on tools, both

DOI: $10.1187 /$ cbe.12-08-0127

Address correspondence to: Brian O'Shea (oshea@msu.edu).

(C) 2013 B. O'Shea et al. CBE-Life Sciences Education (C) 2013 The American Society for Cell Biology. This article is distributed by The American Society for Cell Biology under license from the author(s). It is available to the public under an AttributionNoncommercial-Share Alike 3.0 Unported Creative Commons License (http:/ / creativecommons.org/licenses/by-nc-sa/3.0).

"ASCB ${ }^{(\mathbb{}) " ~ a n d ~ " T h e ~ A m e r i c a n ~ S o c i e t y ~ f o r ~ C e l l ~ B i o l o g y ~}{ }^{(\mathbb{R}) " ~ a r e ~ r e g i s-~}$ tered trademarks of The American Society for Cell Biology. experimental and theoretical, that require a strong quantitative and physics background to understand and use effectively. In response to this, recent reports have called for curricular changes that more fully integrate the physical sciences into the education of life sciences students and those who want to pursue careers in medical fields (American Association for the Advancement of Science [AAAS], 2009; Association of American Medical Colleges-Howard Hughes Medical Institute, 2009; National Research Council, 2010). One specific example of this is the document Vision and Change in Undergraduate Biology Education: A Call to Action, published by the AAAS (2009), which argues for a set of core concepts that all undergraduate biology students should understand, including "structure and function" and "pathways and transformations of energy and matter." The former concept requires students to understand the physics behind the functioning of structural components of living organisms, and the latter concept requires students to understand how the laws of thermodynamics govern the flow of energy in living systems. More broadly, the Vision and Change document outlines a set of core competencies that all biology students need to develop, which include the "ability to use quantitative reasoning," the 
"ability to use modeling and simulation," and the "ability to tap into the interdisciplinary nature of science" (AAAS, 2009, pp. 14-15). The first two competencies tap directly into the core strengths of physics as a discipline-namely, its quantitative, model-oriented nature-and the latter offers an opportunity to more directly tie together the physical and life sciences. To this end, some forays have been made toward tighter integration of biological principles into physics courses and vice versa, the most notable being the National Science Foundation (NSF)-funded, interdisciplinary National Experiment in Undergraduate Science Education (NEXUS) program at the University of Maryland (see Redish, 2012); however, these projects are in their infancy.

In this paper, we present outcomes from redesigning an introductory physics course sequence at Michigan State University (MSU) to directly address the needs of life sciences students. During the 2011-2012 academic year, we implemented a Physics of the Life Sciences curriculum in a physics course sequence whose population is primarily made up of undergraduates majoring in the life sciences. This curriculum is based on a draft textbook that takes a novel approach to teaching physics, focusing on the way in which fundamental physical principles dictate the form and function of organisms. In addition to a new textbook, substantial revisions were made to the homework, recitation, and laboratory components of the course to emphasize the relationship between physics and the life sciences and to help the students learn to apply physical intuition to life sciences-oriented problems. We primarily discuss changes made in the first semester of the course sequence, in which a broader array of both quantitative and qualitative assessments are available to analyze than in the second semester of the sequence. In this paper, we attempt to address the question "Can we measurably and positively change conceptual understanding and attitudinal outcomes of our student population by using discipline-specific physics examples?"

This paper is organized as follows. In Context of the Curricular Changes, we discuss the context within which these curricular changes were made and the population of students taking the course. The New Curriculum describes the new curriculum that has been implemented. Assessment of Changes describes the means we used to assess the changes to the first semester of the course sequence, with the results being described in Results. Finally, in Discussion and Future Work, we discuss the results and some further changes that we will make in response to these results.

\section{CONTEXT OF THE CURRICULAR CHANGES}

The course used in this project is the first semester of an introductory calculus-based physics course taught in Lyman Briggs College (LBC), a residential college within MSU. LBC is one of MSU's flagship programs, and is an undergraduate living-learning community whose main goal is to bridge the sciences and humanities through interdisciplinary teaching and learning (LBC, 2012). The faculty of LBC is composed of scientists, mathematicians, and humanities scholars whose work focuses on the history, philosophy, and sociology of science. Faculty members in LBC often participate in interdisciplinary teaching and research and encourage their students to do the same. This is done most visibly through the NSF- funded Bridging the Disciplines with Authentic Inquiry and Discourse program (BRAID; Luckie et al., 2012). The first iteration of this program attempted to explicitly make connections between LBC-taught first-year courses, specifically, biology and chemistry; later incarnations included other courses and disciplines (Luckie, 2012).

LBC typically accepts students only as freshman and takes approximately 625 students per year on a first-come, firstserved basis. Enrollment into the college occurs when students accept an offer from MSU and declare LBC to be their college of choice. After enrolling in LBC, students can choose any number of majors within the college, including one of the sciences; mathematics; computer science; and the history, philosophy, and sociology of science (HPS). All students in the college are required to take a core set of classes, including two-semester introductory biology, chemistry, and physics sequences, as well as calculus and several HPS courses. Students who decide they wish to major in other disciplines, or to pursue the equivalent non-LBC science major, may opt to transfer to another college within MSU if they choose. Although a range of disciplines are available to LBC students, the majority of students who graduate from the college major in the life sciences ( $85 \%$ in 2011), and more than $80 \%$ of students in a given graduating class plan to pursue postbaccalaureate education of some kind, with popular choices being medical school, veterinary school, and graduate school in medical and life sciences-related fields. In the 2010-2011 academic year, the composition of the incoming class of 627 students was $55 \%$ female, $22 \%$ minority students, and $8 \%$ outof-state students (with no distinction made in this category between domestic and foreign students). Due to attrition, the total size of the college in the 2010-2011 academic year was 1865 students, with the most recent graduating class being composed of 293 students, $55 \%$ of whom were female. While attrition from LBC is substantial (more than $50 \%$ of students starting at LBC do not finish their degree in the college), the vast majority of students beginning at LBC remain at the university and complete their degrees-roughly $90 \%$ of students who start in LBC as freshmen end up graduating from MSU within 6 years.

Most colleges and universities typically offer two introductory physics course sequences: a three-semester, calculusbased sequence targeted at engineers and physical scientists, and a two-semester, algebra-based sequence for all other students who are required to take physics. MSU offers these two options to all students; however, LBC offers a third option. This third option is a two-semester, calculus-based physics sequence (LB 273 and LB 274) that is offered only to LBC students. Historically, this course sequence covered approximately the same material as a standard two-semester, algebrabased course (kinematics, dynamics, and a small amount of thermodynamics in the first semester; electricity and magnetism, optics, and "modern physics" in the second semester), albeit in somewhat greater depth. This course sequence has three 50-minute lectures per week, as well as one 3-hour "hands-on session," which is a combination of recitation and laboratory activities with content that is integrated tightly with the lectures and reading material. The lecture sections range from 50 to 130 students, and the hands-on sessions have up to 24 students, with students working in either pairs or groups of four under the supervision of two undergraduate learning assistants, who are older LBC students who took the 
course in previous years (for an example of a similar program, see Otero et al., 2010). A variety of active-learning techniques are used in both the lecture and hands-on sections, including think-pair-share conceptual and quantitative questions (King, 1993) and individual and small-group problem solving in lecture, and concept-focused Washington Group physics tutorials (McDermott and Shaffer, 2001), context-rich physics problems (Heller, 2012), and inquiry-driven laboratory exercises in the hands-on sessions. Preclass questions on the reading and weekly homework are assigned and graded using the LON-CAPA course management system (Kortemeyer et al., 2008).

LBC students have the option of either taking the twosemester, calculus-based sequence offered by LBC or enrolling in one of the two university-wide, and much more traditional, introductory physics course sequences. Over the course of several semesters, a steady decrease in enrollment in the LBC physics course sequence was observed, with the majority of these students choosing to take MSU's algebrabased, introductory physics course sequence instead. In an attempt to understand this phenomenon, LBC faculty asked LBC students who had taken one of the three physics course sequences to fill out an anonymous survey explaining their choice of courses. Overwhelmingly, it was reported that the LBC physics sequence was more rigorous and required more effort of the students than the university's algebra-based sequence, and that the acquisition of the highest possible grade was considered to be much less certain. Furthermore, many students expressed a lack of understanding as to why they should have to take physics and how it might help them in the future. Taken together, this caused many LBC students to opt to take the course perceived as being the least amount of work with the highest certainty of getting the best possible grade.

\section{THE NEW CURRICULUM}

In response to the challenges described in the preceding section-namely, falling course enrollment and students' lack of understanding of the relevance of physics to the life sciences and their career plans- the LBC physics faculty decided to more explicitly make connections between physics and the life sciences. After considering the options, we settled on the the draft textbook Physics of the Life Sciences, by Professor Timothy McKay (University of Michigan). McKay's textbook is different from other nominally similar texts (such as Newman, 2008) in several intriguing ways. This textbook takes a novel approach to teaching physics to life sciences majors by explicitly focusing on how physical principles dictate the shape, size, and structure of organisms, with an emphasis on physical scaling laws, such as the relationship between an object's mass and its surface area. Such scaling laws are touched upon repeatedly to explain such disparate phenomena as the systematic way that metabolic rates vary with animal size, the convergent evolution that is observed in flying creatures in different animal kingdoms, and how the behavior of diffusion at the atomic level dictates the size of single-celled organisms and the requirement that large animals have circulatory systems in addition to lungs or gills. In addition, McKay's textbook spends substantially more time on thermodynamics, statistical mechanics, and fluid statics and dy-

\section{Modeling a bicep curl: how much force must your bicep muscle exert at this point in the curl?}

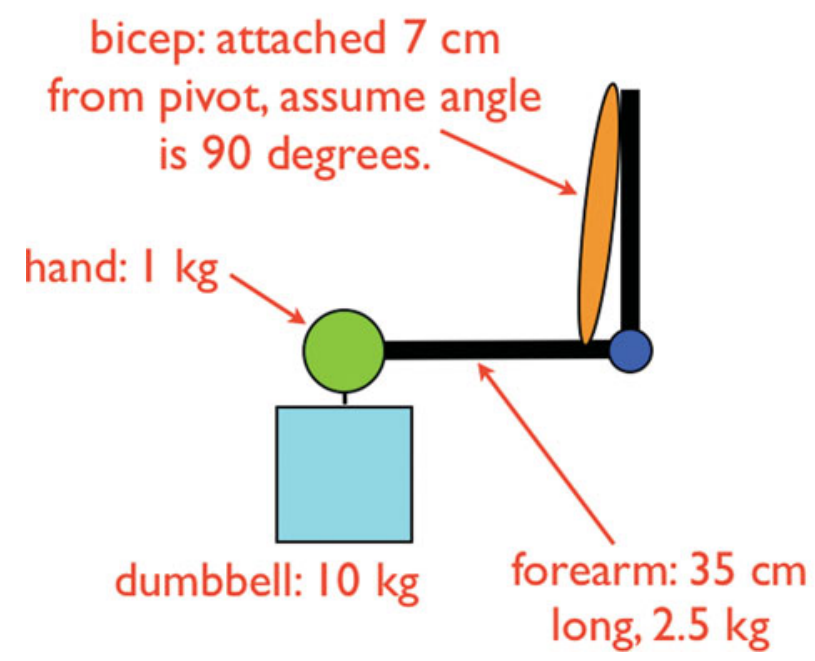

Figure 1. Diagram of a human arm lifting a dumbbell in a bicep curl. Students are asked to work in small groups to model the armdumbbell system at this point in the exercise and must use several physics concepts (force, torque, and free-body diagrams) simultaneously.

namics than a standard introductory physics textbook, and less time on kinematics and dynamics-changes that reflect the needs of life sciences students. Finally, McKay's book is calculus-based, whereas the standard physics texts targeted toward life sciences majors are algebra-based (e.g., Newman, 2008), and thus lack the mathematical rigor that we desire, or are true biophysics textbooks, which are aimed at much more advanced students who have already taken basic physics and have a much more extensive background in mathematics.

Other aspects of the course were also modified in support of this new curriculum. The lecture sessions, which were already highly interactive, were modified to include both conceptual and quantitative questions with a life sciences theme, with additional problems explicitly designed to introduce students to the idea of modeling living systems. An example problem given in lecture, used during the section of the course dedicated to understanding forces, is as follows (with the accompanying illustration being shown in Figure 1):

Consider a person holding a dumbbell in their hand and performing a bicep curl. When doing this, they hold the dumbbell by their side, and then, without moving their elbow, slowly lift the weight until it is touching their chest. Consider the diagram shown in Figure 1, which shows the position of the weight, bones, and bicep when the person's forearm is being held horizontal. Given the numbers provided, how much force must this person's bicep muscle exert at this point in the curl?

Problems of this sort require a multistep solution and the understanding of several physics tools and concepts, including free-body diagrams, force, and torque. They also tie into a broader discussion of locomotion-muscles only pull, and 
cannot push, so the construction of both invertebrates with exoskeletons and vertebrates are designed around this fundamental constraint.

We also developed a substantial number of new homework problems within the LON-CAPA course management system that explicitly use the physics principles discussed in lecture and in the reading materials to bring life sciences students to an understanding of a wide array of situations that may be of interest to them. We also sought to ensure that these problems are authentic to the disciplines involved (e.g., Watkins et al., 2012) and emphasize the conceptual thinking that the students will find most useful in their careers (e.g., Nichparenko, 1985). For example, problems created for the first semester of the course sequence explore the following topics:

- How the response to pressure differs in healthy bones and osteoporotic bones, with experimental data from Thurner et al. (2005) used to demonstrate why people with arthritis are more prone to breaking their bones when falling than are healthy individuals.

- How the shape and size of animals result in different freefall velocities (e.g., why an elephant has a higher free-fall velocity than a mouse, and why mice can therefore fall from great heights without harm).

- How the diffusion rate of the ATP molecule within the body sets fundamental limits on the duration of high-intensity exercise.

- The relative ability of organisms of different sizes to generate heat and dissipate it (and why small animals tend to have problems with maintaining a high enough body temperature, and large animals are prone to overheating).

- The relative blood pressure in wider areas of a blood vessel (i.e., aneurysms) and narrower areas of a blood vessel compared with the mean, and the effects that this change in pressure may have on the stability of the circulatory system (explaining why aneurysms and blocked arteries are of such grave concern).

Similar problems were developed for our hands-on sessions, during which students were asked to work in small groups to model, for example, the forces and torques on the human arm when lifting a heavy object, and for both the midterm and final exams, which were entirely free response. A representative free-response exam question from the first midterm exam is:

Imagine that your evil physics professor lured you into a machine that shrunk you to be $1 \mathrm{~cm}$ tall (isomorphically, of course).

After a bout of maniacal laughter, he got distracted and left you on top of a standard-height table. Would you be able to safely jump from the table to the floor? Why or why not?

In your shrunken state, you adopt a carpenter ant as a means of transportation. You manage to get back into the machine, which transforms you back to your normal size. As a side effect, your trusty steed is now almost six feet tall! What is at least one scaling-related challenge that a human-sized carpenter ant would face?

Correctly solving this exam question requires students to couple their understanding of the scaling law that controls the relationship between an object's mass, surface area, and the cross-sectional area of its limbs. These factors dictate the relative importance of forces such as gravity and air resistance, as well as the ability of a creature's limbs to support its body.

\section{ASSESSMENT OF CHANGES}

In this section and the next, we focus on assessment of, and outcomes from, only the first course in the two-semester sequence, in which a broader array of both quantitative and qualitative assessments are available to us. The reasons for this are primarily practical: interviews focusing on the first semester of the course sequence (offered in the Fall) were conducted over winter break or at the start of the Spring term, when the course was still fresh in the students' minds. This was effectively impossible to do at the end of the Spring term.

The changes made to this course were assessed in several ways. We administered two standard physics education research instruments-the Force Concept Inventory (FCI; Halloun and Hestenes, 1985a,b) and the Maryland Physics Expectations Survey (MPEX; Redish et al., 1997, 1998)—as both pre- and posttests during the first semester of the course sequence. The FCI is a 28-question, multiple-choice test used to assess students' conceptual understanding of Newton's laws; it is widely used in high school and college-level mechanics courses (Hake, 1998). The MPEX is an attitudinal survey that assesses students' understanding of physics as a discipline and their expectations for and attitudes about taking a physics course; it asks a series of questions with Likertscale responses. The MPEX has also been administered to a large number of $\mathrm{PhD}$-possessing physicists, thus allowing the quantification of expert-like attitudes. Both the FCI and the MPEX are standard and widely used physics education research instruments that allow us to quantitatively compare learning gains and student attitudes both with previous iterations of our own courses (in which both instruments have been administered for several consecutive years) and with courses at other universities. Students were given full participation credit for taking both the FCI and MPEX pretests, with no penalty on the FCI for incorrect answers or rewards for correct ones. Given that the MPEX is an attitudinal survey, with no "correct" answer, this issue is not relevant. Students received participation credit for both the FCI and MPEX posttests, although they were given a small amount of extra credit if their score was above $70 \%$ for the FCI. While in principle this might bias our results, this reward has been given every semester that the FCI has been administered in LBC, which reduces the chance of it affecting our comparisons in a nonsystematic way.

Qualitative outcomes were assessed in several ways. An anonymous midsemester survey was administered during the seventh week of class. This survey was open-ended, and asked questions such as "What's going well in the class?" and "What needs improvement?" During the last week of class, students were asked to fill out a paper-based version of the Student Assessment of Learning Gains (SALG; Seymour et al., 2000), which, in addition to the standard complement of multiple-choice questions, asked for open-ended feedback on the course. Students were prompted to think about different aspects of the course with five questions: 
1. Which topics did you like the most, and which the least?

2. Which topics seemed most relevant to you, and why?

3. Which aspects of the course structure (lecture, hands-on sections, online homework, help room, etc.) were most and least useful to you, and why?

4. Do you have any other suggestions as to how we might improve the course?

5. Do you have any suggestions as to how I might improve my teaching?

Students were not given any external incentive to complete either of these surveys, though we note that the SALG was administered during class time to enhance the response rate. Also, students are strongly encouraged to give extensive written feedback in all LBC courses, so response rates are typically high -71 students out of 77 (92\%) filled out the multiplechoice component of the end-of-semester evaluation, and 61 of those $(86 \%)$ provided substantial written feedback as well.

In addition to the written surveys, a total of 15 students were interviewed by one of the coauthors of this paper (L.T.), and anonymized transcripts of these interviews were provided to the primary author (B.O.'S.) for analysis (described in more detail in Affective Outcomes). The interviewer (L.T.) has no association with the class outside of the interview process, and students were informed both in the invitation email and prior to the interview that course staff (in particular, the primary author of this paper and primary course instructor, B.O.'S.) would not know whether they participated in the interviews or not. Of the 15 interviewees, nine were selected at random from the course roster, with three students apiece chosen from the top, middle, and bottom third of the course grade distribution. The remaining six students were randomly selected from a pool of 16 students suggested by the course faculty as being particularly likely to have insightful thoughts on the physics course they had just completed. The interviews were conducted between the end of the Fall semester final exam session and the second week of the Spring semester, and were done using an interview protocol that combined a pregenerated set of questions with in-depth follow-up questions to probe student responses more fully. The pregenerated questions targeted student motivations ("Why did you take this class?"), connections the students made to course content ("What did you particularly connect to in this course? Were there any specifically memorable assignments, discussions, or lectures?"), and impact of the course's learning goals on the students' thinking ("How did the topics discussed during the semester tie together?") and on their lives outside their physics course ("Did you talk about things that were covered in class with other people who were not enrolled in the course? If so, could you give me an example?"). In addition to posing the pregenerated questions, the interviewer followed up on students' comments to get more detail or to clarify vague statements. The interview transcripts were examined by the primary author (B.O.'S.) for common themes and for correlation of student responses with other factors, such as the students' grades in the course and their majors.

\section{RESULTS}

In Fall 2011, LB 273 (Physics I) was cotaught by two of the authors of this article (B.O.'S. and W.B.), with B.O.'S. run- ning the lecture and LON-CAPA components of the course, and W.B. running the hands-on component. Seventy-five students were enrolled in the course at the beginning of the Fall semester, and 77 students completed the course. The increased number over the semester is due to a handful of students switching from the university's algebra-based course into the LBC physics course during the first week of the semester and a few students dropping the course. The course was composed of $\sim 54 \%$ females and $\sim 46 \%$ males, with the vast majority of students (73 of the 77 who finished the semester) self-identifying as life sciences majors.

\section{Conceptual Outcomes}

Quantitatively, student outcomes paint a positive picture. The pretest mean FCI score was $42.3 \%( \pm 4.88 \%$; $n=75)$, which is substantially higher than previous semesters' pretest mean score of $33-36 \%$ ( $\pm 2 \%$; Cruz et al., 2010). Given that the LBC student population has not evolved substantially over the past few years (in terms of demographics and standardized test scores), this argues that the students choosing to take the course in Fall 2011 were, on average, of higher quality than average. We speculate that this is due to a combination of two factors: less-prepared students systematically choosing to take alternate physics courses that are perceived to be easier (as suggested by the results of our anonymous survey from the summer before the course was offered), and better-prepared and more intellectually curious students systematically choosing to take the course specifically due to the advertised Physics of the Life Sciences focus. The mean posttest FCI score was $61.6 \%( \pm 7.02 \% ; n=77)$, compared with $\sim 55 \%( \pm 3.5 \%)$ in earlier semesters (Cruz et al., 2010). The standard method to measure gain in such situations is to consider the normalized gain, $<\mathrm{g}>$. This is defined as the improvement in students' understanding of Newton's laws compared with the maximum possible gain available to them, or:

$$
<g>=(\text { post }- \text { pre }) /(100-\text { pre })
$$

where "post" is the posttest score, and "pre" is the pretest score. Our normalized gain is $\langle\mathrm{g}\rangle=0.334 \pm 0.0381$, which is statistically indistinguishable from previous implementations of this course (Cruz et al., 2010), in line with introductory physics courses at other universities that use research-based interactive engagement techniques, and substantially higher than traditional, lecture-based courses (Hake, 1998; Caballero et al., 2012). Overall, our students' performance on the FCI indicates that implementing the new curriculum has done no harm to our students' conceptual understanding of physics compared with the previous curriculum, although we have not substantially increased their conceptual understanding of Newton's laws compared with previous semesters. Given that the goal of our curricular change was to enable students to more explicitly make connections between physics and the life sciences, and not to increase conceptual understanding of Newtonian mechanics, this result is quite acceptable. In fact, given that somewhat less time is spent on Newton's laws, having learning gains that are comparable to previous semesters is actually quite encouraging!

In addition to the FCI, we administered the MPEX as both a pretest and posttest. Prior work shows that it is typical for students' attitudes toward physics to become substantially 
Table 1. Favorable fraction for the six dimensions of student expectations probed by the MPEX

\begin{tabular}{lccc}
\hline \multicolumn{1}{c}{ Cluster } & Favorable pre $(n=69)$ & Favorable post $(n=75)$ & Shift (post - pre $)$ \\
\hline Independence & $0.571 \pm 0.066$ & $0.541 \pm 0.062$ & -0.030 \\
Coherence & $0.559 \pm 0.065$ & $0.608 \pm 0.070$ & +0.021 \\
Concepts & $0.457 \pm 0.053$ & $0.478 \pm 0.055$ & +0.021 \\
Reality link & $0.757 \pm 0.087$ & $0.743 \pm 0.085$ & -0.014 \\
Math link & $0.650 \pm 0.075$ & $0.642 \pm 0.074$ & -0.008 \\
Effort & $0.675 \pm 0.078$ & $0.549 \pm 0.063$ & $-0.126^{\mathrm{b}}$ \\
\hline
\end{tabular}

${ }^{a}$ MPEX administered at the beginning (pre) and end (post) of the Fall 2011 semester. The shift in scores is defined as post - pre. Errors shown are SEs. "Favorable fraction" is defined as the fraction of students whose answers to this cluster of questions are consistent with the answers given by a set of PhD-level university physics faculty interested in reforming their teaching and making it more effective.

${ }^{\mathrm{b}}$ The only statistically significant shift (as determined by a one-way analysis of variance).

more negative after a semester of introductory physics, although this strongly depends on the student population and course in question (Kortemeyer, 2007; Redish et al., 1997). In a typical analysis of the MPEX, questions are grouped into clusters that correspond to student attitudes about independence of learning; understanding of physics as a coherent, consistent framework; conceptual understanding of physics; links to reality; mathematics as a way of representing physical phenomena; and effort exerted toward the class. Furthermore, student surveys are compared with a set of expert answers, which were determined by the MPEX authors by administering the survey to a group of university physics faculty who were committed to reforming their teaching to make it more effective. This expert group provided answers that were strongly consistent (typically at the $90 \%$ level or greater), suggesting that mastery of physics is tied to having or developing the attitudes expressed. Table 1 shows the fraction of students giving favorable answers to the six clusters of questions, both before and after the semester ("favorable" is defined as an answer on the same side of the Likert scale as that of the expert physicists). No statistically meaningful change was observed when comparing MPEX pre- and posttest values for five of the six clusters. We contrast the observed lack of change in Fall 2011 with the MPEX pre- and posttest values observed in previous iterations of this course (taught in Fall 2009 and 2010), in which student MPEX scores become substantially more negative in the "reality link" cluster, meaning that students' belief that the ideas learned in this course are widely applicable to reality actually declined in previous semesters (see Kortemeyer, 2007). This is an interesting result-while the students enrolled in this class do not seem to be fundamentally changing their thinking about physics as a discipline and in its connection to "reality," the change in curriculum is not having a negative effect (and in some sense is a positive change compared with the previous curriculum). We note that we do see a substantial negative change in the "effort" cluster, from a favorability fraction of 0.675 before the semester to 0.549 afterward. This is typical of the vast majority of physics courses, both traditional and reformed, and is believed to be due to the change from "precourse optimism" to "postcourse reality checks" (Redish et al., 1997). We also note that our precourse "favorable" ratings are somewhat higher than in previous semesters (see Table II in Kortemeyer, 2007), which may indicate that the student population enrolled in the class has a more positive overall attitude toward physics (or our specific course sequence) than the student popula- tions enrolled in previous iterations of the course. This may introduce a bias in the results of our survey.

A final quantitative indicator of the impact of the curricular changes we have implemented is year-over-year course enrollment in the LBC physics sequence. Prior to the Fall 2011 semester, course enrollment had experienced a steady decline, to a minimum of 77 students in Fall 2011. Nearly all of the students enrolled in Fall 2011 (74 of 77 students, or 96\%) enrolled in the second semester of the course sequence during Spring 2012, with an additional 11 students transferring to the LBC physics course sequence from elsewhere (the transfer of such a large number of students into the course sequence is unprecedented-typically, enrollment drops by roughly $5 \%$ between consecutive semesters of physics 1 and 2 in all of the physics course sequences at MSU). Of this population of 85 students, a total of 81 finished the course. In comparison, as of the beginning of the Fall 2012 semester, 124 students are enrolled in LB 273-a 61\% increase from the previous year's enrollment of the same class. It seems that the LBC students are "voting with their feet" and may see the value of this curricular change. Of course, this is just one of many possible explanations for the observed increase. Other explanations of this outcome include, but are not limited to, the possibility that LBC students' perceptions of their options with regards to introductory physics courses may have changed from previous semesters, making the LBC physics sequence relatively more attractive. Further data are needed to determine the actual impact of the course revisions on student enrollment.

\section{Affective Outcomes}

Results from our qualitative assessments were quite illuminating. As described in the preceding section, LBC uses a paper-based version of the SALG as an end-of-term course evaluation instrument, and a total of 61 students out of 77 finishing the course (79\%) gave anonymous, open-ended feedback prompted by a series of questions, including "Which topics did you like the most, and which the least? Which topics seemed most relevant to you, and why?" (See Assessment of Changes for a complete listing of questions.) No prompting with regard to the life sciences focus of the course was given.

The SALG free-response section and the interviews were examined for themes using the following methodology. First, the primary author of this paper (B.O.'S.) read through all of the SALG responses and interviews twice, shuffling the order between each reading. During this first cursory 
examination, several broad themes were tentatively identified for each set of documents, and a more detailed examination of each SALG form and interview was then conducted, using these themes as a template. We note that two separate sets of themes were used: no attempt was made to ensure consistency between the SALG written responses and the interviews. During this reexamination, it became clear that the tentative themes found were too highly articulated and that what originally appeared to be different categories of comments were really the same sort of comment expressed in somewhat different ways (e.g., "I absolutely hated the course pack because... [followed by a list of reasons]" and "The course pack could be improved" were originally counted as separate categories, but were later judged to be the same thematic issue expressed with different levels of substance). After the reexamination was conducted, a final pass through the data was made to double-check the categorization. One person was responsible for all categorization, and thus no reliability of categorization was possible (though some assistance was requested from colleagues for various categorizations, this was not done in a systematic way).

Examination of the SALG free-response items showed that several themes were present, with the two primary ones being 1) ability to connect physics to a life sciences-related topic, and 2) dissatisfaction with some aspect of the course pack used for the course. These themes were analyzed more closely to identify substantial comments and also minor/incidental comments. Twenty-five of the 61 students who provided written feedback specifically and positively mentioned the life sciences focus of the course, with 18 making in-depth comments, such as "I really like the scaling law stuff ... it helped me understand why you can have whales in the ocean but not on land!" and "This seems like it's going to be super-useful in the future when I take physiology." A further seven students made minor positive comments, such as "I like how the examples relate to my major" or "I liked the life sciences examples." The majority of students interviewed (eight of 15) enjoyed the focus of the course, with a substantial number (four students) indicating that, despite early skepticism, they found that the course was ultimately quite interesting. Six students mentioned that they saw connections between the course, their own interests, and other scientific disciplines, such as chemistry and biology. "Dr. Timothy McKay, from University of Michigan, he said in the beginning of the course that physics is the science that would explain chemistry, that would explain biology ... When he talks about the thermodynamics ... I did feel like the things I was learning from chemistry were really integrated into the physics, and physics. Learning what he taught me actually gave me a deeper understanding of that." The interviewed students clearly liked the focus of the course, and thought that the life sciences-focused examples provided by the textbook, in lecture, and in the hands-on sessions helped to keep their interest. In general, the interview subjects liked the "big picture" aspect of the course-namely, using the laws of physics to understand the structure and behavior of organisms-and found that to be very memorable and relatable to their interests. Simply liking something does not imply understanding, of course; however, when asked to provide specific and physically correct examples connecting the physics they learned to their interests, nine of the 15 students interviewed were able to do so. "Suspensory ligaments and how far they can stretch, going back to springs, how far they can stretch before they break. That was memorable to me. As an animal science major, when I come up with a circumstance where an animal has come up lame or something, I always think of that because I was able to make the connection." Two students interviewed, who self-identified as nonlife sciences majors, specifically mentioned the life sciences focus of the course in a negative light, however, complaining that there were too many life sciences examples. "We're not all pre-meds, you know?"

A significant number of interviewees (four of 15) mentioned that, due to their interest in the material, they talked about these examples with people who were not enrolled in the course. "I have talked to my parents a little bit probably relating the blue whale stuff. Those things definitely excite me, the general concepts, like I said, the overall class of relating physics to life sciences." A few students (three of 15 interviewees) mentioned that, in addition to speaking to others about physics-related ideas, they found themselves thinking about physics concepts in everyday situations, such as walking on ice, driving a car, or watching an animal run.

A large number of students expressed concern or displeasure with the draft textbook (24 of 61 students on the SALG; seven of 15 students in interviews); however, additional comments (eight on the SALG form and four from the interviews) indicated that this was not due to the focus of the textbook, but rather due to the lack of practice problems beyond the assigned homework problems and also the confusing explanations of some phenomena. "I didn't like the course pack. You had to find the information. There was just a lot of useless fluff but once you found the information the course pack did help, it just took two or three times of reading it to actually get there." In fact, the life sciences focus of the textbook was a significant highlight of the student feedback, with four of 15 interviewed students bringing up the life sciences theme of the textbook in a positive light and without prompting. "That's the one thing I liked about the textbook was in every chapter they tried to relate it to like a life sciences, like in biology or something. Just using diffusion and using that as far as like the pulmonary region in the lungs and stuff was effective for me." It seems clear from this feedback that the published version of this textbook, containing example problems and supplemental homework problems, would substantially help the students' acceptance of the text.

\section{Instructor Outcomes}

In addition to feedback relating to students enrolled in the course, it is perhaps worth considering the impact that the new curriculum has had on the course personnel, particularly the instructors (and B.O'S.). When the decision was made to switch to this curriculum, it sparked extensive and positive discussions with other LBC science faculty about the changes that were going to be made, the examples used, and the possible connections that could be explicitly drawn between the LBC physics sequence and the other introductory courses taught in the college (including continuing discussions about how to integrate the physics courses into the LBC BRAID curriculum). Over the course of the semester, faculty from other disciplines took the time to observe the class, examine course materials, and provide valuable feedback about the prior knowledge of the LBC students who had taken their courses before enrolling in physics. Furthermore, as part of 
the process of digesting the new textbook and creating life sciences-focused homework and exam problems, B.O'S. had the opportunity to think about a range of subjects that he had not been exposed to in nearly two decades, and which have been quite personally satisfying to explore. Finally, the clear enthusiasm of the students toward the life sciences-focused examples used in the class has been profoundly refreshing and has led to a much higher rate of positive student-faculty interactions than in previous semesters.

\section{DISCUSSION AND FUTURE WORK}

Converting the LBC introductory physics sequence from a fairly standard two-semester physics curriculum to one that focuses on the needs of life sciences students has been a significant positive step. Students' conceptual understanding of Newtonian mechanics has not been negatively impacted, based on quantitative metrics such as the FCI. Related to this, the course sequence covers most of the same topics, and with the same level of rigor as in previous iterations of the course, but now includes additional topics that were not previously covered. The material that has been removed (primarily relating to kinematics and rotational motion) is of limited utility to our target population, while the content that has been expanded, including thermodynamics and fluid dynamics, is much more relevant to our students' needs in future courses. Furthermore, the qualitative data from interviews and surveys show the majority of students enrolled in the class clearly enjoyed the new focus of the course sequence and stated that it helped to maintain their interest over the course of the semester and to make connections between physics and their own majors. The significant year-over-year enrollment increase also suggests that students in LBC feel that the knowledge they have gained is worth the additional effort (with an alternate interpretation being that students' perceptions of alternative courses have changed in the favor of our course, thus driving a climb in an enrollment). Overall, the experiment was a success.

There are several ways in which this study is limited. Primarily, we caution that the results presented in this paper may be biased due to the student population participating in the course. As discussed in the Results, the students enrolled in LB 273 in Fall 2011 had substantially higher pretest scores on the FCI than students in previous semesters $(\sim 42 \%$ compared with 33-36\%). This may indicate that the students in the Fall 2011 semester had a higher academic ability than previous student populations, which may have positively influenced their receptiveness to the new curriculum. Alternately, the FCI results may simply represent more prior experience with physics among our student population.

Other limitations include the limited period of data collection (a single semester) and the choice of this time period (the first time the course was taught, when student and instructor enthusiasm might be affected most strongly). The results may not generalize to the second-semester course, which focuses on topics in electricity, magnetism, and modern physics, and which was not evaluated in a similar manner. Similarly, we have made no attempts in this course or in previous courses to systematically measure students' understanding of topics that are more central in the reformed course (e.g., thermodynamics and fluid flow) or core skills (e.g., problem solving).
This is due in part to time constraints, but primarily because no standardized assessment instruments exist for these topics and skills.

Adoption of this physics curriculum has been deemed successful in LBC and will likely continue to be used in the future with the full support of the faculty and college administration. This curriculum could be easily adopted by other institutions, although we note that the order and emphasis of topics is substantially different from that of a more standard course, which could be uncomfortable for some faculty (particularly physics faculty who are uninterested in or uncomfortable with the life sciences) and may require significant rethinking of hands-on instructional components; purchasing new lab and/or demonstration hardware; and an investment in time to create a substantial quantity of life sciences-focused recitation, homework, and exam problems (with the last three items likely being the biggest initial barrier to adoption). Implementation of this curriculum requires support at the faculty and departmental level at the very least, particularly in largeenrollment courses with multiple instructors and a great deal of inter-section coordination, as is common at many large R1 institutions. Given that the list of topics is qualitatively similar to a more standard introductory physics curriculum, it seems unlikely that new courses would need to be created or that substantial negative impact on courses that have introductory physics as a prerequisite would be felt. The reforms discussed in this paper are relatively easy to insert into an existing curriculum without changing other courses. If both life sciences and physical science faculty were supportive, however, one could imagine implementing a much more comprehensive set of reforms, including enforcing a much more extensive set of course prerequisites, including chemistry and biology (e.g., see the University of Maryland's Project NEXUS [Redish, 2012]). In this circumstance, one of the largest barriers to adoption would be the discomfort and/or hostility that many physicists appear to feel when asked to teach about life sciences topics that they may not have personally engaged with in many years, coupled with a similar set of feelings on the part of life scientists who are asked to engage with physics topics. This initial discomfort can likely be mitigated by careful choice of a faculty cohort and by encouragement and support at the college and university level.

As with any curriculum that is being used for the first time, there is substantial room for improvement in the next iteration of the course:

- The draft textbook was generally well received but needs to be supplemented with additional practice problems until a published textbook is available.

- Additional homework problems need to be developed, particularly multipart problems that explore the physical principles behind specific living systems.

- Appropriate laboratory exercises need to be developed. At present, the recitation-like component of the hands-on section is strongly coupled with the new curriculum, but our laboratory exercises are relatively standard.

- The introductory chemistry and biology course sequences that are taught in LBC are already tightly coupled through the BRAID program, and it would be logical to more formally integrate the physics course sequence into this program. 
Of the points listed above, the last one has the potential to have the most impact on students' long-term success. Projects of an interdisciplinary nature are becoming more prevalent in the life sciences and in medicine, and the ability to apply multiple conceptual lenses (i.e., disciplinary viewpoints) to a problem is a very useful skill set. Giving students the opportunity to develop such skills, perhaps by some sort of extended project, may help to further increase student involvement and learning.

\section{ACCESSING MATERIALS}

All materials created for this course, including lectures, homework, and exam problems; hands-on session materials; solutions for all materials; and a guide containing implementation advice are available for no charge. Please email the author directly at oshea@msu.edu to receive copies of these materials.

\section{ACKNOWLEDGMENTS}

The authors acknowledge support for this effort from MSU's Office of Faculty and Organizational Development and LBC through the Lilly Teaching Fellowship. We thank Punya Mishra for his invaluable advice and guidance and Alicia Alonzo, Deborah DeZure, Cori FataHartley, Elizabeth Gire, Elizabeth Simmons, Kendra Spence Cheruvelil, and Steven Wolf for useful discussions. We are very grateful to Timothy McKay for generously providing us with his draft textbook and other course materials. Finally, we are grateful to two anonymous referees for providing suggestions that have greatly improved the quality of the manuscript.

\section{REFERENCES}

American Association for the Advancement of Science (2009). Vision and Change in Undergraduate Biology Education, Washington, DC. http:/ /visionandchange.org (accessed 31 August 2012).

Association of American Medical Colleges-Howard Hughes Medical Institute (2009). Scientific Foundations for Future Physicians, Washington, DC: AAMC. www.aamc.org/scientificfoundations (accessed 31 August 2012).

Caballero M, et al. (2012). Comparing large lecture mechanics curricula using the Force Concept Inventory: a five thousand student study. Am J Phys 80, 638-644.

Cruz E, O'Shea BW, Schaffenberger W, Wolf S, Kortemeyer G (2010). Tutorials in introductory physics: the pain and the gain. Phys Teach $48,453-457$.

Hake R (1998). Interactive-engagement versus traditional methods: a six-thousand-student survey of mechanics test data for introductory physics courses. Am J Phys 66, 64-74.

Halloun I, Hestenes D (1985a). Common sense concepts about motion. Am J Phys 53, 1056-1065.
Halloun I, Hestenes D (1985b). The initial knowledge state of college physics students. Am J Phys 53, 1043-1055.

Heller K (2012). UMPERD Context-Rich Problems, Minneapolis: University of Minnesota. http://groups.physics.umn.edu/ physed/Research/CRP/crintro.html (accessed 31 August 2012).

King A (1993). From sage on the stage to guide on the side. Coll Teach 41, 30-35.

Kortemeyer G (2007). The challenge of teaching introductory physics to premedical students. Phys Teach 45, 552-557.

Kortemeyer G, Kashy E, Benenson W, Bauer W (2008). Experiences using the open-source learning content management and assessment system LON-CAPA in introductory physics courses. Am J Phys 76, 438-444.

Luckie D (2012). BRAID Project, East Lansing: Michigan State University. www.msu.edu/ luckie/braid/Site/Whats_BRAID/ Whats_BRAID.html (accessed 31 August 2012).

Luckie D, Bellon R, Sweeder RD (2012). The BRAID: experiments in stitching together disciplines at a big ten university. J STEM Educ 13, 6-14.

Lyman Briggs College (2012). Mission, Vision and Values, East Lansing: Michigan State University. www.lymanbriggs.msu.edu/ mission.cfm (accessed 31 August 2012).

McDermott LC, Shaffer PS (2001). Tutorials in Introductory Physics, Upper Saddle River, NJ: Prentice Hall.

National Research Council (2010). BIO2010: Transforming Undergraduate Education for Future Research Biologists, Washington, DC: National Academies Press, 191.

Newman J (2008). Physics of the Life Sciences, New York: Springer, 736

Nichparenko S (1985). Premed physics: what and why. J Coll Sci Teach 14, 391-394.

Otero V, Pollock S, Finkelstein N (2010). A physics department's role in preparing physics teachers: the Colorado learning assistant model. Am J Phys 78, 1218.

Redish E (2012). Project NEXUS UMCP, College Park: University of Maryland-College Park. http://umdberg.pbworks.com/w/page/ 44091483/Project\%20NEXUS\%20UMCP (accessed 31 August 2012).

Redish E, Steinberg R, Saul J (1997). The distribution and change of student expectations in introductory physics. AIP Conf Proc 399, 689-698.

Redish E, Steinberg R, Saul J (1998). Student expectations in introductory physics. Am J Phys 66, 212-224.

Seymour E, Wiese D, Hunter A, Daffinrud SM (2000). Creating a better mousetrap: online student assessment of their learning gains. Paper presented at the National Meeting of the American Chemical Society, San Francisco, CA, March 27, 2000.

Thurner P, et al. (2005). High-speed photography of human trabecular bone during compression. Mater Res Soc Symp Proc 874, L1.2.1L1.2.6.

Watkins J, Coffey E, Redish E, Cooke T (2012). Disciplinary authenticity: enriching the reforms of introductory physics courses for lifescience students. Phys Rev ST Phys Educ Res 8, 010112. 